\title{
ARTICLE
}

\section{The effect of Parylene coating on the surface roughness of PMMA after brushing}

\section{Short Title: Parylene coating affects PMMA surface roughness}

\author{
Mariana Santos ${ }^{1}$, Steven Soo ${ }^{2}$, Haralampos Petridis ${ }^{3}$ \\ 1 Postgraduate student, \\ 2 Lecturer, \\ 3 Senior Lecturer, \\ Prosthodontics Unit, Department of Restorative Dentistry, \\ UCL, Eastman Dental Institute, \\ London, WC1X 8LD, UK
}

Corresponding Author:

Dr. Haralampos Petridis

Prosthodontic Unit

Department of Restorative Dentistry

UCL Eastman Dental Institute

256 Gray's Inn Road,

London WC1X 8LD, UK

Tel: 0044(0)2034561250

e-mail: c.petridis@ucl.ac.uk

\begin{abstract}
Objectives

Acrylic resins, used in the manufacturing of different types of intra-oral prostheses, are vulnerable to colonization by microorganisms which potentially endanger the general health of the prostheses wearers. The aim of this study was to investigate the influence of a novel coating (Parylene) on surface roughness of Poly-methyl-methacrylate (PMMA) samples after simulated cleansing using an electric toothbrush and two brushing media (paste and pumice).

Methods

Fifty six square PMMA samples were fabricated. Half of the samples were coated with a uniform 10 $\mu \mathrm{m}$ coating of Parylene. All samples were subjected to simulated brushing with either paste or pumice. Changes in surface roughness were measured with a laser noncontact profilometer and compared between groups. Scanning electron microscopy (SEM) and Raman spectroscopy were utilized for surface visualization and analysis.
\end{abstract}

Results

In the coated samples, the mean surface roughness remained the same before and after brushing in the Paste group 2.69 $\mu \mathrm{m}(\mathrm{SD}=0.92$ and $\mathrm{SD}=0.87$ respectively), and increased from $3.73 \mu \mathrm{m}(\mathrm{SD}=1.25)$ to $5.05 \mu \mathrm{m}(\mathrm{SD}=1.40)$ in the Pumice Group. In the uncoated samples, the mean surface roughness increased from $4.45 \mu \mathrm{m}(\mathrm{SD}=0.92)$ to $6.73 \mu \mathrm{m}$

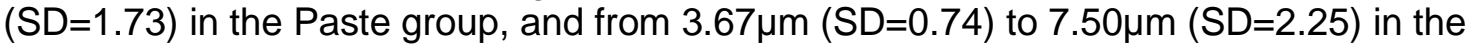
Pumice Group. Differences between the coated and uncoated groups were statistically significant $(p<0.05)$. The surface analyses revealed that the coating remained adhered to the PMMA, although signs of partial detachment were noticed in the Pumice Group. 
Conclusion

The Parylene coating resulted in a reduction of surface roughness of PMMA after brushing procedures.

Clinical Significance

Parylene appears to maintain a low surface roughness of PMMA after abrasion by brushing.

Keywords: Parylene, Acrylic, Roughness, Coating, Brushing, PMMA

\section{Introduction}

Acrylic resins are used widely in fixed and removable prosthetic rehabilitations. Poly-methylmethacrylate (PMMA) is an acrylic resin commonly used in the fabrication of denture bases. PMMA surfaces are susceptible to indentation by hard objects and easy to abrade. These phenomena contribute to an increase in surface roughness and wear of the acrylic, leading to a favourable environment for biofilms to accumulate. Colonization of dentures by microorganisms like Candida albicans and Streptococcus oralis in the form of biofilms has been associated with denture stomatitis [1,2] and frequently associated with general disease in diabetic patients, hospitalised

Page $303-->$

populations and other immunocompromised patients. Methicillin-resistant Staphylococcus aureus (MRSA) has also been reported as present in dentures and its difficult erradication may facilitate the spread of infection in hospital environments [3].

In 1997 a clinical study by Bollen et al. [4] established that the threshold roughness (Ra) for plaque retention of intra oral materials was $0.2 \mu \mathrm{m}$. Several studies have shown that brushing action causes increased surface roughness in acrylic materials $[5,6,7,8,9]$. The subsequent colonisation of a rough surface by microorganisms is also a well-documented fact $[10,11,12]$ as the micro crevices created by the surface degradation serve as a shelter and a reservoir for microorganisms.

Roughness parameters can be analyzed either in two (2D) or in three (3D) dimensions with 3D profilometers gaining popularity as they can provide a broader and more comprehensive image of a material's surface characteristics and support specific imaging software [13]. Various measurements of surface roughness can be obtained, with $\mathrm{Ra}$, which is the arithmetic average height measurement, being frequently used as a universal roughness parameter for general quality control [13]. Profilometers can be used to compare the same surface in different times and after different levels of wear and are classified either as contact or non-contact. The latter will not involve any part of the instrument physically touching the surface being analyzed, whereas the former involve a stylus running in direct contact with the surface of the sample while recording its texture [14].

Parylene is the commercial patented name of a family of organic polymers called poly(paraxylylenes) formed by means of Vapour Deposition first observed by Szwarc, in 1947 [15]. The production of Parylene films starts with the pyrolytic decomposition at $600^{\circ} \mathrm{C}$ of $\mathrm{p}$ cyclophane, a dimer composed of two $p$-xylylene molecules, into its respective molecules $[16,17]$. The substance obtained this way is a vapour of monomer that, if injected into a deposition chamber at room temperature, rather than going back to its original dimeric powder form, produces instead a transparent, strong, highly crystalline polymer film.

Parylenes exhibit a very high level of biocompatibility and very low toxicity and are therefore, indicated for medical uses $[18,19]$. They are nowadays widely used in medical sciences as a coating material for metallic implants [20], for covering biological micro-electromechanical 
systems (BioMEMS) [21] and encasing implantable neural electronic devices [22]. Its use in oral appliances has shown some possible advantages, such as the creation of a beneficial microbial ecological shift, protection against corrosion [23] and the increase in the fracture resistance of dental ceramics [24]. Although its adhesion to some materials may not be easily achieved, several methods can be used to bypass this and achieve a reliable adhesion [18, 22].

The aim of this study was to compare the changes in surface roughness after brushing cycles with pumice or paste between PMMA samples coated with Parylene and control uncoated samples. The null hypothesis was that no statistically significant difference in surface roughness change would be detected after brushing between samples coated with Parylene compared to uncoated samples.

\section{Materials and Methods}

Sample Size determination:

Sample size was calculated using nQueryAdvisor® (Statistical Solutions, Cork, Ireland) and data from previous published studies. A final sample size of 14 samples per group was decided based on the calculation that it would allow for a 95\% power to detect a difference in mean roughness of $1.5 \mu \mathrm{m}$ assuming that the common standard deviation is 1.00 , using a two-group t-test with a 0.05 two-sided significance level.

\section{Sample Fabrication:}

Square samples of PMMA (Diamond $D \circledast$ Heat Cure Denture Resin, Keystone Industries, New Jersey, USA) measuring $15 \times 15 \mathrm{~mm}$ were fabricated after being processed in a large two-part metal flask. Every square was analysed under an optical microscope (Mantis Elite ${ }^{\circledR}$ Vision Engineering, Surrey, England) at 10x magnification and any samples showing gross surface defects were rejected. Fifty six samples were randomly divided into 4 equal stacks and attributed a group number. The first and second groups (test groups) were coated with Parylene whereas the third and fourth groups (control groups) were left uncoated. The samples in the test and control groups were subsequently brushed with either paste or pumice. The samples in the test groups were handled as follows: The samples were packaged in moisture tight sealed bags and sent to be coated with Parylene to Specialty Coating Systems Ltd (SCS), Surrey, England. The target thickness of the Parylene coating was $10 \mu \mathrm{m}$ on all sides. Control of the coating thickness achieved after the 2 cycles was ascertained by using coupons placed inside the chamber.

\section{Experimental Testing:}

A custom-made brushing apparatus was designed and built for this experiment. An electric toothbrush (Oral B Triumph 5000®, Procter and Gamble, Surrey, England) was fixed in a horizontal position over a plastic holder and secured to a flat surface. The brush operated at 48,800 oscillations per minute. A weight of $200 \mathrm{~g}$ was placed on top of the brush's head. A small gypsum well was used to retain the samples and prevent pumice or paste escaping during brushing. The brush was fixed so that the brush head position was consistent between samples and was fully charged each time. The brush head was replaced for a new one every 5 samples within the same group. Two different brushing media were used: a pumice slurry (Skillbond®, Skillbond Direct, High Wycombe, England) and a commercially available Toothpaste (Oral B Pro-Expert@, Procter and Gamble, Surrey, England). To simulate one year of wear, a total of 20 minutes brushing per sample was undertaken [25]. Two milliliters of each slurry were placed on the well over the sample with a plastic pipette before the brushing commenced. Every 2 minutes the slurries were replenished while the brush kept rotating to compensate for the slurry lost with the oscillation/vibration of the brush head. The slurry used was always taken from the same depth in the container after agitation so that the average amount of abrasive particles was 
Page $804-->$

similar in every cycle. At the end of the brushing cycle the samples were rinsed under running water, dabbed dry using a soft paper tissue and left to dry at room temperature.

Roughness Measurements:

The initial (before brushing) and final (after brushing) surface roughness measurements (arithmetic mean height - Ra) were taken from each sample using a Laser Profilometer (ProScan $1000 \AA$ Scantron, Sommerset, England). Surface roughness measurements were also obtained for seven uncoated PMMA samples, before coating in order to analyze the effect of Parylene coating on initial surface roughness.

Two areas of $3 \times 3 \mathrm{~mm}(9 \mathrm{~mm} 2)$ on each sample were chosen where abrasion was predicted (initial $\mathrm{Ra}$ ) or observed (final $\mathrm{Ra}$ ) and an average roughness value of each area was measured and calculated. For each area the Profilometer scanned 600 lines, $0.005 \mathrm{~mm}$ away from each other to obtain the final $3 \times 3 \mathrm{~mm}$ area. The Ra measurements were obtained by choosing 5 points diagonally down the area and recording the $\mathrm{Ra}$ of the 2 lines that intercepted at each point. Obvious aberrations in surface topography in the $9 \mathrm{~mm} 2$ area scanned were ignored and no point with lines crossing those areas was read except when impossible to avoid in highly roughened areas after testing

Visual Surface Analysis:

A Scanning Electron Microscope (SEM) (JEOL JSM 5410 LVSEM, JEOL Ltd, Herts, England) was used to visualize the surface topography of control and experimental samples. One sample from each group and 2 untested samples (one coated and one uncoated) were coated with sputtered gold and visualised at x50, x200 and x1000 magnification. An attempt was made to focus on a representative area and increase the magnification while remaining on the same area.

Surface Molecular Analysis:

After the brushing procedure all samples from the Test Groups were analysed using a Laser Raman Spectroscope (Labram 300, Horiba Jobin Yvon) to detect the presence of the Parylene coating. This spectroscope uses a HeNe $(633 \mathrm{~nm})$ laser. Additionally, one sample that had not been subjected to abrasion cycles was also analysed to serve as control. In each sample an area measuring $100 \times 100 \mu \mathrm{m}$ was chosen in the most abraded surface present.

\section{Statistical Analysis:}

The mean roughness values before and after the Parylene coating before brushing, were analysed using the Paired t-test. Multivariable Linear Regression analysis was undertaken analyze the effect of coating and brushing medium on the final Ra after adjusting for the initial Ra. The computer software SPSS® (Version 20, IBM, Portsmouth, UK) was used for the statistical analysis and production of graphs. The interaction between coating and brushing medium was included as a co-variant in the regression analysis. The assumptions underlying the regression analysis were checked by study of the residuals. A significance level of 0.05 was used for the hypothesis testing. 


\section{Results}

Roughness measurements:

The mean roughness values, before and after brushing, in the 4 experimental groups are shown in Table 1.

In the Control Groups (28 samples of uncoated PMMA) there was an increase in mean final roughness of $51.24 \%$ compared to the initial $\mathrm{Ra}$ in the Paste Group (Fig.1) and an increase of $104.36 \%$ in the Pumice Group. All samples in the Control

Page $805-->$

Groups showed an increase in average surface roughness after the brushing procedure. In the Test Groups (28 samples of Parylene coated PMMA) there was no change in mean final Ra scores compared to the initial Ra in the Paste Group (Fig.1) and there was a 34.67\% increase in the Pumice Group. Seven samples in the Test Group showed a decrease in mean surface roughness ( 4 in the Paste group and 3 in the Pumice group) after the brushing cycle.

Seven samples from the Test Group were scanned before being sent for coating. This was done to analyse the change in roughness granted by the Parylene coating alone. Surface roughness decreased in all the samples after coating from a mean $\mathrm{Ra}$ of $3.830 \mu \mathrm{m}$ before $(\mathrm{SD}=1.132)$ to $2.750 \mu \mathrm{m}$ after $(\mathrm{SD}=1.084)$.

Statistical analysis of roughness results:

To check the assumptions underlying the regression analysis, the residual values were plotted and revealed a normal distribution and constant variation. The statistical analysis of the results revealed that the coating significantly affected the Final $\mathrm{Ra}(\mathrm{p}<0.005)$, with its presence leading to reduced final Ra values by a mean of $2.8 \mu \mathrm{m}(95 \% \mathrm{Cl}: 1.9-3.7 \mu \mathrm{m})$ after adjusting for the brushing medium and initial $\mathrm{Ra}$. The brushing medium also exhibited a statistically significant effect on the roughness with the pumice increasing the roughness by $1.5 \mu \mathrm{m}(\mathrm{p}<0.005,95 \% \mathrm{Cl} 0.6-2.4 \mu \mathrm{m})$ when compared to paste, after adjusting for the presence of the coating and the Initial $\mathrm{Ra}$. The mean roughness values before and after the Parylene coating were analysed using the Paired t-test and revealed a significant change in roughness $(p<0.05)$.

SEM analysis:

The results of the SEM analysis are depicted in Figure 2. There were evident differences between the various groups, as explained in the legend.

Raman Analysis:

After the brushing procedure all samples from the Test Groups were analysed using the Raman Laser Spectroscope. Notable

Page 806 - - >

peaks at the regions of $308,865,1015,1204,13361441$ and $1608 \mathrm{~cm}-1$ are characteristic of the Parylene C film [26]. Parylene peaks were found in all the samples brushed with paste (Fig.3). In the samples brushed with pumice only one sample demonstrated an absence of Parylene, with only peaks representing PMMA visible on the spectrum analysis. 


\section{Discussion}

Poly-methyl methacrylate is one of the oldest and most commonly used materials in dentistry. The inherent limitations of PMMA make it susceptible to bacterial and fungal colonization when used for the fabrication of intraoral prostheses [1-3]. The aim of this study was to investigate whether the coating of PMMA samples with Parylene would have an effect in reducing surface roughness after simulating 1 year of brushing with either paste or pumice. A reduction in roughness could possibly lead to a lower risk of microbial colonization $[10,11,12]$. Furthermore, the use of this coating in the manufacturing of removable or fixed PMMA protheses could potentially increase the life-span of these devices by delaying the loss of material and the increase in surface roughness due to abrasion from chewing and brushing procedures.

The results of this study showed that the Parylene coating of PMMA led to a statistically significant reduction in mean Ra values before and after the brushing cycles. Samples coated with Parylene showed a lower increase in mean surface roughness after brushing (no change in the Paste Group and $34.67 \%$ for the Pumice Group) when compared to the uncoated samples (51.24\% in the Paste Group and 104.36\% in the Pumice Group). The linear regression analysis established these differences as statistically significant with $\mathrm{p}<0.05$. The null hypothesis was, therefore, rejected.

All Ra measurements presented with values above the $0.2 \mu \mathrm{m}$ described by Bollen et al. [4] as the threshold for bacterial adhesion, showing that even coated samples in this study could be susceptible to microbial colonization. This might have been due to the fact the samples did not undergo any polishing procedure before testing. Nevertheless, the beneficial effect of coating demonstrated could lead to further development and testing of different parameters, such as coating thickness and initial surface polishing, in order to approach the $0.2 \mu \mathrm{m}$ threshold. These parameters are currently being tested in other experimental designs.

The increase in surface roughness of PMMA after the brushing cycles is consistent with most of the literature on this subject $[4,8,27,28]$. It is not possible to compare the absolute surface roughness values of this study with other studies as there are differences in methodology (processing and polishing methods) and surface analysis using different scanners. In this study a laser non-contact profilometer was used. Other types, such as contact profilometers could have been used, but the main disadvantage of the latter devices is the fact that the contact of a stylus against the easily abraded surface of the acrylic could cause the roughness measured to be skewed due to the plowing effect of the stylus [29]. Denture patients are advised to use some form of denture cleaning powder during cleansing procedures. Paste was used as a medium in this study as various surveys [30, 31] have reported its frequent use by patients. Pumice, although not regularly used for denture cleansing, was chosen as a second medium in order to subject the coating to extreme brushing and abrasion conditions. The Raman analysis showed the persistence of the Parylene coating after brushing, proving an efficient adhesion of the polymer to the acrylic substrate. The SEM analysis showed that the coating retained its integrity after brushing with paste, but showed signs of deterioration after the pumice treatment. This aggressive, irregular texture might also

Page 807 - - >

explain the higher Ra values obtained. Clinically, this would translate to the necessity for a less aggressive cleansing protocol. This should be the subject of a future investigation on the adequacy of cleansing procedures.

There were some limitations to this study. Only the Ra values were recorded for each sample, a fact which limited the extent in which these results could be used to characterise 
the acrylic surface. $\mathrm{Ra}$ is the arithmetic average height measurement, and was used in this study as it is considered a universal roughness parameter for general quality control [13]. A different parameter like the $\mathrm{Rz}$, the ten-point height measurement, could have also been used as it is considered more sensitive to high peaks and deep valleys in the surface of a material. The results, in $\mathrm{Ra}$, were nonetheless comparable with other studies on this subject, including the study by Bollen et al [4]. Also the Ra values demonstrated a relatively high standard deviation (from 0.87 to $2.25 \mu \mathrm{m}$ ). This relates to the variation present in the initial roughness readings and the variability inherent to the fact that the acrylic samples, although made from the same 2 acrylic sheets, presented with microscopic irregularities that the processing procedure could not bypass. However, the sample size and randomization procedures were adequate to allow for valid conclusions to be drawn and the normal distribution of the data was demonstrated by the residuals plotted. Thermo cycling was not used in this study. Although this process produces an artificial ageing effect that may affect the bond to the substructure, previous studies have shown that Parylene coatings were very resistant to prolonged contact with physiological fluids and even sterilization procedures [32, 33]. Based on this literature, it was decided not to include this parameter in this experimental design in order to isolate the material's reaction to the brushing alone.

The results of this study show some positive effect of the use of Parylene as coating for intraoral prostheses made of PMMA, regarding surface roughness. More experiments are currently being conducted in order to further develop this technique.

\section{Conclusions}

The coated samples showed a statistically significant lower change in roughness after the brushing procedures compared to the uncoated samples.

The coated samples brushed with paste showed no change in roughness before and after the brushing procedures.

The samples brushed with pumice in both groups showed a higher roughness after brushing than the ones brushed with paste. 


\section{Acknowledgements}

Based on a dissertation submitted to UCL as partial fulfillment of the requirements for the MSc degree in Conservative Dentistry

Mr Aaron Clark (SCS Coatings, Surrey, England) for the kind offer to coat the samples

Mr Colin Clark, Dr Nicola Mordan, Dr Graham Palmer for their technical advice, and Dr Aviva Petrie for the guidance in the statistical analysis (Eastman Dental Institute, UCL)

ORAL B, Procter and Gamble, Surrey, England for the kind provision of the electric toothbrush.

\section{References}

1. Johnson CC, Yu A, Lee H, Fidel PL, Noverr MC. Development of a contemporary animal model of Candida albicans-associated denture stomatitis Using a novel intraoral denture system. Infection and Immunitty, 2012;80:1736-1743.

2. Charman KM, Fernandez P, Loewy Z, Middleton AM. Attachment of Streptococcus oralis on acrylic substrates of varying roughness. Letters Applied Microbiology, 2009;48:472-477.

3. Smith AJ, Jackson MS, Bagg J. The ecology of Staphylococcus species in the oral cavity. Journal of Medical Microbiology, 2001; 50:940-946.

4. Bollen CML, Lambrechts $P$, Quirynen M. Comparison of surface roughness of oral hard materials to the threshold surface roughness for bacterial plaque retention: A review of the literature. Dental Materials, 1997;13:258-269.

5. Heath JR, Davenport JC, Jones PA. The abrasion of acrylic resin by cleaning pastes. Journal of Oral Rehabilitation, 1983;10:159-175.

6. Oliveira LV, Mesquita MF, Henriques GEP, Consani RLX, Fragoso WS. Effect of polishing technique and brushing on surface roughness of acrylic resins. Journal of Prosthodontics, 2008;17:308-311.

7. Freitas-Pontes KM, Silva-Lovato $\mathrm{CuH}$, Paranhos HFO. Mass loss of four commercially available heat-polymerized acrylic resins after toothbrushing with three different dentifrices. Journal of Applied Oral Sciences, 2009;17:116-121.

8. Machado AL, Giampaolo ET, Vergani CE, Pavarina AC, da Silva Lopes Salles D, Jorge $\mathrm{JH}$. Weight loss and changes in surface roughness of denture base and reline materials after simulated toothbrushing in vitro. Gerodontology, 2012;29:e121-e127.

9. Li L, Finnegan MB, Ozkan S, Kim Y, Lillehoj PB, Ho CM, Lux R, Mito R, Loewy Z, Shi W. In vitro study of biofilm formation and effectiveness of antimicrobial treatment on various dental material surfaces. Molecular Oral Microbiology, 2010;25:384-390.

10. Yamauchi M, Yamamoto K, Wakabayashi M, Kawano J. In vitro Adherence of microorganisms to denture base resin with different surface texture. Dental Materials Journal, 1990;9:19-24.

11. Morgan TD, Wilson M. The effects of surface roughness and type of denture acrylic on biofilm formation by Streptococcus oralis in a constant depth film fermentor. Journal of Applied Microbiology, 2001;91:47-53. 
12. Pereira T, Cury A, Cenci MS, Rodrigues-Garcia RCM. In vitro Candida colonization on acrylic resins and denture liners: Influence of surface free energy, roughness, saliva, and adhering bacteria International Journal of Prosthodontics, 2007;20:308-310.

13. Gadelmawla ES, Koura MM, Maksoud TMA, Elewa IM, Soliman HH. Roughness parameters. Journal of Materials Processing Technology, 2002;123:133-145.

14. Poon CY, Bhushan B. Comparison of surface roughness measurements by stylus profiler, AFM and non-contact optical profiler. Wear, 1995;190:76-88.

15. Szwarc M. Some remarks on the ch2= benzene $=\operatorname{ch} 2$ molecule. Discussions of the Faraday Society, 1947;2:46-49.

16. Gorham WF. A new, general synthetic method for the preparation of linear Poly-pxylylenes. Journal of Polymer Science A Polymer Chemistry, 1966;4:3027-3039.

17. Smalara K, Gieldon A, Bobrowski M, Rybicki J, Czaplewski C. Theoretical study of polymerization mechanism of $p$-Xylylene based polymers. Journal of Physical Chemistry A. 2010;114:4296-4303.

18. Bienkiewicz J. Plasma-enhanced parylene coating for medical device applications. Medical Device Technology, 2006;17:10-11.

19. Wolgemuth L. Parylene: Surface modifier for implanted devices. European Cells and Materials Journal, 2012:23:8.

20. Kaminska M, Okroj W, Szymanski W, Jakubowski W, Komorowski P, Nosal A, et al. Interaction of parylene $\mathrm{C}$ with biological objects. Acta of Bioengineering and Biomechanics, 2009;11:19-25.

21. Chang TY, Yadav VG, De Leo S, Mohedas A, Rajalingam B, Chen C-L, et al. Cell and protein compatibility of parylene-C surfaces. Langmuir, 2007;23:11718-11725.

22. Hassler C, von Metzen RP, Ruther P, Stieglitz T. Characterization of parylene $C$ as an encapsulation material for implanted neural prostheses. Journal of Biomedical Material Research B, 2010;93:266-274.

23. Wilson M, Kpendema $\mathrm{H}$, Noar JH, Hunt N, Mordan NJ. Corrosion of intra-oral magnets in the presence and absence of biofilms of Streptococcus sanguis. Biomaterials. 1995;16:721725.

24. Teixeira EC, Piascik JR, Stoner BR, Thompson JY. Zirconia-parylene multilayer thin films for enhanced fracture resistance of dental ceramics. Proceedings of the Institution of Mechanical Engineers H Journal of Engineering in Medicine, 2009;223:897-902.

25. Lewis R, Dwyer-Joyce RS. Interactions between toothbrush and toothpaste particles during simulated abrasive cleaning. Proceedings of Institution of Mechanical Engineers $\mathrm{J}$ Journal of Engineers in Tribology, 2006;220:755-765.

26. Jakabovič J, Kováč J, Weis $M$, Haško $D$, Srnánek R, Valent $P$, et al. Preparation and properties of thin parylene layers as the gate dielectrics for organic field effect transistors. Microelectronics Journal, 2009;40:595-597. 
27. Haselden CA, Hobkirk JA, Pearson GJ, Davies EH. A comparison between the wear resistance of three types of denture resin to three different dentifrices. Journal of Oral Rehabilitation, 1998;25:335-339.

28. Harrison Z, Johnson A, Douglas CWI. An in vitro study into the effect of a limited range of denture cleaners on surface roughness and removal of Candida albicans from conventional heat-cured acrylic resin denture base material. Journal of Oral Rehabilitation, 2004;31:460-467.

29. Wassel RW, McCabe JF, Walls AWG. (1994) Wear characteristics in a two-body wear test. Dental Materials, 1994;10:269-274.

30. Polyzois G. Denture cleansing habits. A survey. Australian Dental Journal, 1983;28:171173.

31. Dikbas I KT, Calikkocaoglu S. Investigation of the cleanliness of dentures in a University Hospital. International Journal of Prosthodontics, 2006;19:294-298.

32. Beshchasna N, Adolphi B, Granovsky S, Braunschweig M, Schneider W, Uhlemann J, Wolter KJ. Influence of artificial body fluids and medical sterilization procedures on chemical stability of Parylene C. In: Electronic Components and Technology Conference (ECTC) Proceedings 60th, Las Vegas 2010:1846-1852.

33. Hsu J-M, Kammer S, Jung E, Rieth L, Normann AR, Solzbacher, F. Characterization of Parylene- $C$ film as an encapsulation material for neural interface devices. In: Dimov S, Menz W and Toshev Y, editors. Third International Conference on Multi-Material Micro Manufacture. Borovets, 2007. 
Table 1 - Surface roughness values before and after brushing in the four experimental groups

\begin{tabular}{|c|c|c|c|}
\hline $\begin{array}{c}\text { Experimental } \\
\text { Groups }\end{array}$ & $\begin{array}{c}\text { Brushing } \\
\text { Medium }\end{array}$ & Mean Initial $\mathbf{R}_{\mathbf{a}}$ (SD) & $\begin{array}{c}\text { Mean Final } \mathbf{R}_{\mathbf{a}} \\
\text { (SD) }\end{array}$ \\
\hline $\begin{array}{c}\text { Control Group } \\
\text { Uncoated } \\
\text { PMMA }\end{array}$ & Paste & $4.45 \mu \mathrm{m}(0.92)$ & $6.73 \mu \mathrm{m}(1.73)$ \\
\cline { 2 - 4 } & Pumice & $3.67 \mu \mathrm{m}(0.74)$ & $7.50 \mu \mathrm{m}(2.25)$ \\
\hline $\begin{array}{c}\text { Test Group } \\
\text { Parylene } \\
\text { Coated PMMA }\end{array}$ & Paste & $2.69 \mu \mathrm{m}(0.92)$ & $2.69 \mu \mathrm{m}(0.87)$ \\
\cline { 2 - 4 } & Pumice & $3.73 \mu \mathrm{m} \mathrm{(1.25)}$ & $5.05 \mu \mathrm{m} \mathrm{(1.40)}$ \\
\hline
\end{tabular}

$\mathrm{SD}=$ Standard Deviation; $\mathrm{Ra}=$ Arithmetic Surface Roughness; $\mathrm{PMMA}=$ Poly methyl methacrylate 


\section{CAPTIONS}

Table 1 - Surface roughness values before and after brushing in the four experimental groups

$\mathrm{SD}=$ Standard Deviation; $\mathrm{Ra}=$ Arithmetic Surface Roughness; $\mathrm{PMMA}=$ Poly methyl methacrylate

Figure 1 - Graph showing the roughness values before and after brushing with Paste in the Parylene and Control groups

Figure 2 - SEM images (x1000 magnification) of the samples after testing a) a coated sample after brushing with Paste: a smooth surface is present with particles from the paste visible on the surface; $b$ ) an uncoated sample after brushing with Paste: $a$ roughened surface with streaks and surface degradation is present; c) a coated sample after brushing with Pumice: a degraded surface is present with signs of flaking and detachment of the Parylene coat, no streaks are present; d) an uncoated sample after brushing with Pumice: the surface presents streaks and obvious surface degradation

Figure 3 - Raman analysis showing the spectrum obtained from a sample brushed with paste (lower line) and the control coated un-brushed sample (upper line) 